\title{
Geophysical and geotechnical data fusion for protection dikes characterization
}

\author{
Théo Dezert ${ }^{1}$, Sérgio Palma Lopes ${ }^{1}$, Yannick Fargier ${ }^{2}$, Christophe Vergniault ${ }^{3}$ and Jean-Robert Courivaud ${ }^{4}$ \\ ${ }^{1}$ Université Gustave Eiffel, GERS, GeoEND, 44344 Bouguenais, France \\ ${ }^{2}$ Université Gustave Eiffel, GERS, RRO, 69675 Bron, France \\ ${ }^{3}$ Electricité de France, 13090 Aix-en-Provence, France \\ ${ }^{4}$ Electricité de France, 73373 Le Bourget du Lac, France
}

\begin{abstract}
Dike ruptures may lead to disastrous consequences such as loss of lives and economic disasters. To prevent the risk of breakage, special supervision of the hydraulic structure is required. Usual methodologies for the reconnaissance of earthen dikes include complementary geophysical and geotechnical investigation methods. This paper introduces a new methodology to combine data from these two types of information sources, taking into consideration their specificities (respective level of imperfections and spatial distribution). This innovative procedure considers the mathematical belief masses theory and improves the characterization of lithological materials within a real case study. It provides information on the level of conflict between information sources as well as displays a confidence index associated with the results. The considered investigation campaign involves electrical resistivity tomography, multi-channel analysis of surface waves as well as core drilling with particle-size analysis from laboratory testing. The fusion results highlight the capacity of this combination methodology to characterize the lithological sets and to indicate the interfaces' positions as well as associated levels of confidence.
\end{abstract}

\section{Introduction}

Earthen dikes are structures that require special monitoring in order to prevent the risk of rupture that may lead to catastrophic consequences such as human and material losses as well as economic disasters. There are recognized methodologies for the assessment of dikes that include geophysical and geotechnical investigation methods (Fauchard and Mériaux, 2007; Royet et al. 2013).

Geophysical methods are non-intrusive and provide physical information on large volumes of subsoils with high output but with potentially significant uncertainties. These uncertainties are due in particular to the integrative and indirect aspects of the methods as well as to the resolution of the inverse problems. The measured physical parameters are usually not suited to the analysis of failure modes. In a complementary way, geotechnical investigation methods are intrusive and provide much more local information, but also more precise and better suited to the analysis of failure modes. An important issue for the evaluation of protection dikes is to be able to combine the geophysical and geotechnical data, while taking into account their associated uncertainties, inaccuracies and respective spatial distributions (Royet et al. 2013). It is also important to be able to get a subsoil

a Corresponding author: theo.dezert $@$ univ-eiffel.fr

DOI 10.3311/FLOODRisk2020.17.21 representation more relevant and informative than a simple superposition of different physical parameters.

We propose the use of the belief masses theory (Dempster, 1967; Shafer, 1976) and two combination rules for merging geophysical and geotechnical information. In this work, we wish to demonstrate the potential of our fusion methodology using two geophysical investigation methods (Electrical Resistivity Tomography and Multichannel Analysis of Surface Waves) and one geotechnical investigation method (core drillings with particle-size analysis). We demonstrate the ability of our information fusion approach to characterize the lithology of a real earthen canal dike, associating confidence indexes as well as conflict level between information sources.

The results of such a methodology would be highly valuable for decision support and flood risk mitigation. This is a promising and cost-effective approach being assessed on real case studies. It has been developed with the aim of short-term knowledge transfer to any interested user.

First, this paper starts with a presentation of the investigation methods used in the dike survey campaigns as well as their positioning. It follows with the data acquired by each method and with an introduction to the belief masses theory as well as to the two considered combination rules. The characterization of the dike section 
in the framework of belief masses is then displayed for each investigation method respectively. Finally, the fusion results are presented and discussed to highlight the interests and limits of such a methodology.

\section{Study site and investigation methods}

The study site is a canal dike located in the south of France, belonging to EDF (Electricité de France). The investigated section is located on the right bank of the hydraulic work, from Kilometric Point (KP) 10.35 to 12.13. Geologically, two formations are present in this section as well as a NE-SW fault, lowering the western part. Up to KP 10.8 , the dike is mainly based on a more or less marly limestone substratum (lower Cretaceous). Beyond KP 10.8, the terrain is composed of more or less clayey indurated marls. Finally, between KP 11.5 and 12.13 , locally resistant materials (Cretaceous limestones) may be present.

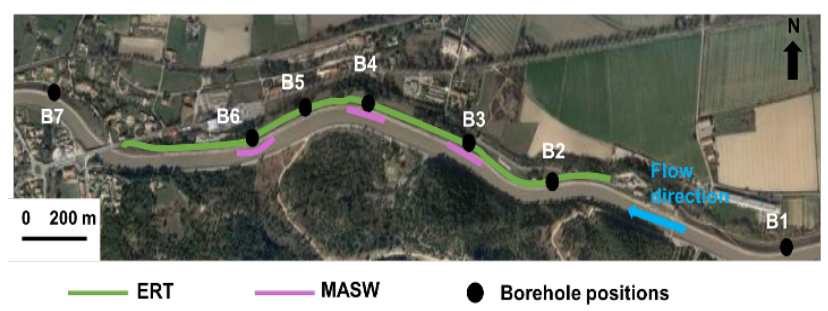

Figure 1. Study site with positions of geophysical and geotechnical investigation campaigns.

On this earthen hydraulic structure, two geophysical campaigns using the Electrical Resistivity Tomography (ERT) and Multi-channel Analysis of Surface Waves (MASW) methods were respectively set up in 2014 and 2017. A geotechnical campaign with core drillings was also carried out in 2016. All investigations were achieved from the dike crest and their positions are specified in Figure 1. The backfill-foundation limit in the dike is displayed in Figures 2 and 3. However, this limit is approximate since the information comes from historical punctual cross sections while we display results in longitudinal section.

\subsection{Electrical Resistivity Tomography}

The basic concept of DC-resistivity methods consists in injecting an electric current of known intensity [A] by means of two "current" electrodes and measuring a voltage [V] between two "potential" electrodes. Data acquisitions are made for many stations (several positions of the potential and the current electrodes). The data acquired are then inverted using an inversion software (Res2DInv ver 3.71.118, Loke, 2013), to reconstruct a complete 2Dsection of electrical resistivity [ $\Omega . \mathrm{m}]$.

The considered device is composed of 48 electrodes with a 5 meters inter-electrode spacing. A "roll-along" process is used to cover the complete length of the profile. The acquisition was carried out considering a DipoleDipole configuration and the position of the profile is displayed in Figure 1.

The inverted electrical resistivity discretized section appears in Figure 2. The depicted values suggest the presence of low resistivities on an area covering more than $500 \mathrm{~m}$ of the levee's length (KP 11.1-11.65). Highly resistive materials seem located between 8 to $16 \mathrm{~m}$ deep (KP 10.4-10.52), near the dike crest (KP 10.55-10.7), between 8 to $24 \mathrm{~m}$ deep (KP 10.8-10.95) and between 6 to $14 \mathrm{~m}$ deep (KP 12-12.1). The interface positions between lithological materials cannot be established precisely.

\subsection{Multi-channel Analysis of Surface Waves}

The MASW method consists in computing shear wave velocities from the study of the dispersion of surface waves. The first stage of the method implies the computation of the Rayleigh dispersion curve from the data and then, the computation of shear wave velocities $(V s)$ after an inversion procedure.

For this geophysical campaign, a homogeneous device of towed streamers was used (shooting every $24 \mathrm{~m}$ ). The acquisitions cover three areas (KP 10.74-10.9, KP 11.111.3, KP 11.5-11.7) within the study dike section (Figure 1). Twenty velocity profiles $\left(\mathrm{m} . \mathrm{s}^{-1}\right)$ of different depths are computed (Figure 3 ) and allow a new specific mesh for this geophysical method.

The shear velocity values suggest low velocities close to the dike crest and higher values below $10 \mathrm{~m}$ depth for the first and third covered areas (KP 10.74-10.9 and KP 11.5-11.7). The second area mainly implies low $V s$ values.

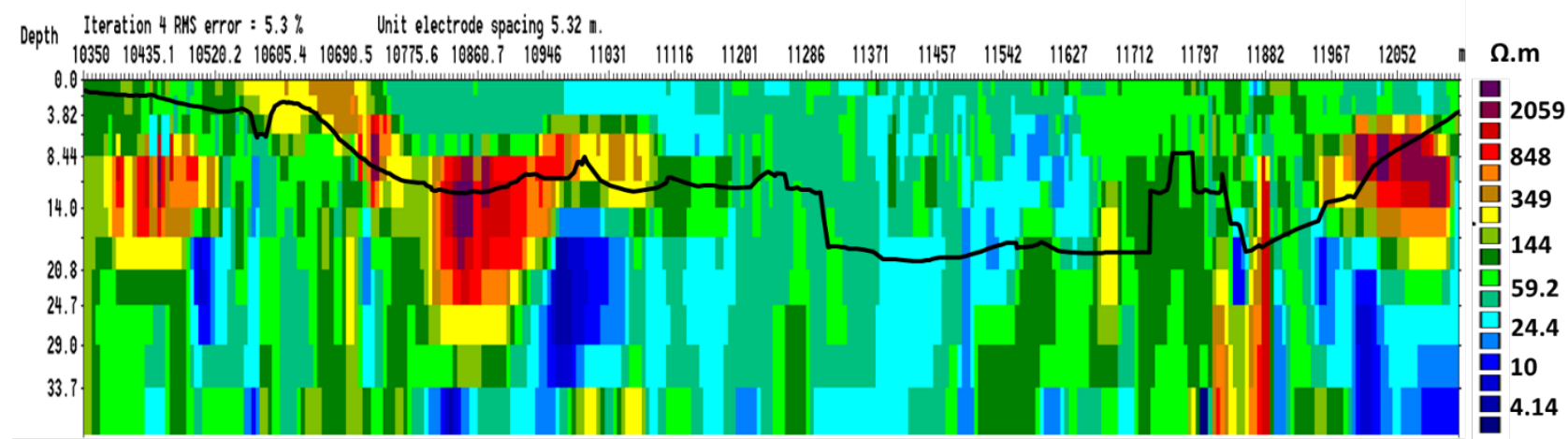

Figure 2. The inverted electrical resistivity $(\Omega . \mathrm{m})$ discretized section obtained between KP 10.35 and KP 12.13. The black line stands for the approximate historical backfill-foundation limit. 


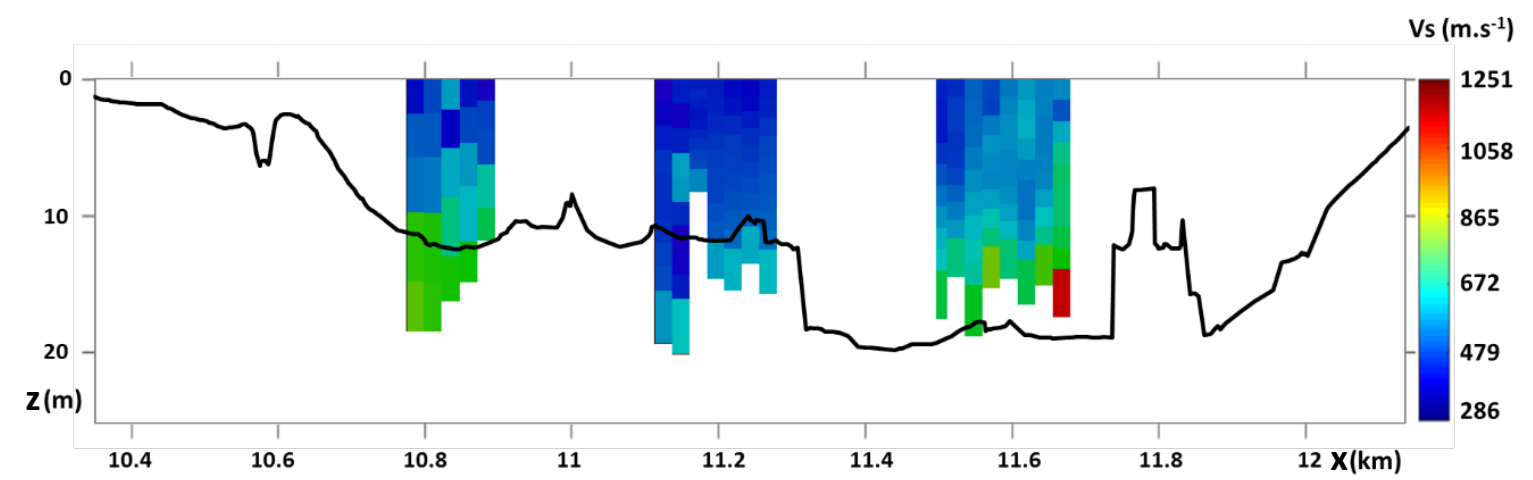

Figure 3. Twenty $V s\left(\mathrm{~m} . \mathrm{s}^{-1}\right)$ profiles obtained after inversion of MASW data in the three discretized investigated areas. The black line stands for the approximate historical backfill-foundation limit.

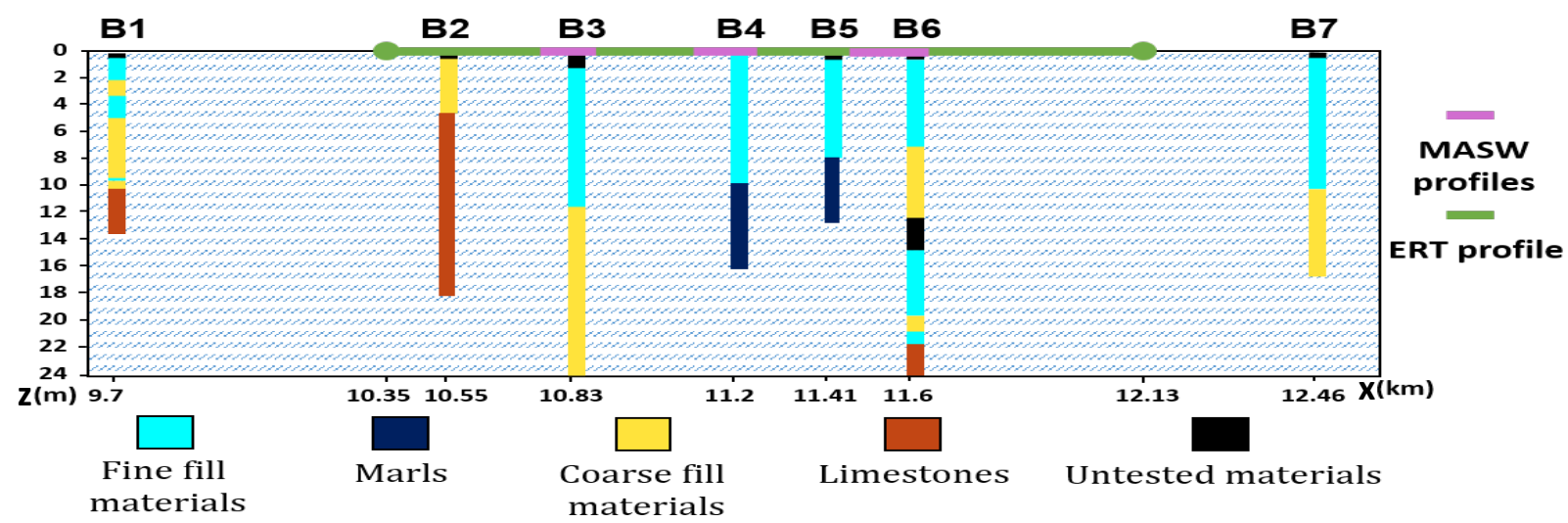

Figure 4. Lithological materials characterized in each of the seven core drillings after particle-size analysis.

\subsection{Drilling cores and particle-size analysis}

Core drillings were carried out on the dike crest, at seven positions, displayed in Figure 1. Boreholes B2 through B6 are located within the ERT profile. The cores were visually identified in the laboratory in order to delineate sections of material that could be considered as belonging to the same particle-size class. Samples were then collected from these cores to perform particle-size analysis tests following the NF P94-056 French standard (1995), except on the most cohesive materials (marls and limestone).

Fine fill materials are discriminated from coarse fill materials using the "Guide des Terrassements Routiers" classification, NF P 11-300 (1992). The lithological characterizations are displayed in Figure 4. Fine fill materials are present close to the surface from $\mathrm{B} 3$ to $\mathrm{B} 7$ and the presence of marls can be observed below for $\mathrm{B} 4$ and B5. Limestone is identified below fill materials in B1, $\mathrm{B} 2$ and $\mathrm{B} 6$ core drillings. It can be pointed out that the lithological basement appears especially at low depths in B2.

\section{Fusion methodology}

\subsection{Belief masses theory and combination rules}

Shafer (1976) introduced the belief masses theory by developing the mathematical theory of evidence inspired by previous works of Dempster (1967). This is why
Shafer's theory is usually referred to as the DempsterShafer theory. The interesting aspect of using belief masses theory lies in its ability to manage information from different sources, associated with their respective levels of uncertainties and inaccuracies. In this work, we consider three information sources: two geophysical (ERT and MASW) and one geotechnical (core drillings). The belief masses theory is also able to assess the conflict (Ø) level between sources, i.e. when the information given by two or more sources are in contradiction. In addition, the theory allows considering the ignorance and incompleteness of the information. Indeed, it is possible to grant credit on all the possible solutions (presence of any of the lithological materials) in order to quantify our ignorance, whereas the probabilistic theory would simply assign an equiprobability to each single solution (each material individually). For the reader eager to learn more, details are present in Martin et al. (2008).

To define and to use the belief masses theory, it is required (i) to set a Frame of Discernment (FoD), (ii) to assign belief masses to the hypotheses of this FoD for each information source, (iii) to choose a fusion rule for merging the information, and (iv) to display a representation of the combined information.

The FoD, $\Theta$, consists of all the possible hypotheses within the problem under concern. The elements of the FoD are exhaustive and exclusive, such as for $n$ hypotheses:

$$
\Theta=\left\{\theta_{1}, \theta_{2}, \ldots, \theta_{n}\right\}
$$


In our problematic, the hypotheses of the FoD correspond to lithological materials. Here, we consider that $\theta_{1}$ stands for fine-grained materials, $\theta_{2}$ stands for marls, $\theta_{3}$ stands for coarse-grained materials, $\theta_{4}$ stands for limestone. We also consider a fifth hypothesis $\theta_{5}$ that is associated to any hypothetical material different from the four previously described, since the elements of the FoD are exhaustive and exclusive. Thus, here, we have:

$$
\Theta=\left\{\theta_{1}, \theta_{2}, \theta_{3}, \theta_{4}, \theta_{5}\right\}
$$

The space of belief mass functions, the set of all subsets of $\Theta$, written $2^{\Theta}$, is fixed by all the disjunctions and by the conflict (Ø) between information sources such that:

$$
2^{\Theta}=\left\{\varnothing, \theta_{1}, \theta_{2}, \theta_{n}, \ldots, \theta_{1} \cup \theta_{n}, \ldots\right\}
$$

The belief mass function $\mathrm{m}$ is attributed to a subset $A$ (defined on $2^{\Theta}$ ) in $[0,1]$ such that, as in the probability theory, the more $m(A)$ tends to 1 and the more the confidence in $A$ is high:

$$
\sum_{A \in 2^{\Theta}} m(A)=1
$$

The approach developed by Smets (1990) allows the assignment of a belief mass to the conflict, so that:

$$
m_{1,2}(\varnothing)>0
$$

Where $m_{1,2}(\cdot)$ denotes the merged belief mass resulting from the fusion of information from sources 1 and 2. The belief mass $m_{1,2}(A)$ resulting from the conjunctive fusion of information from two sources is written:

$$
m_{1,2}(\mathrm{~A})=\sum_{X, Y \subseteq \Theta \mid X \cap Y=\mathrm{A}} m_{1}(X) m_{2}(Y)
$$

With $m_{j}(X)$ the belief mass respectively attributed to hypothesis $X$ by information source $j$. The conflict level between the two considered information sources can therefore be written as:

$$
m_{1,2}(\emptyset)=\sum_{X, Y \subseteq \Theta \mid X \cap Y=\varnothing} m_{1}(X) m_{2}(Y)
$$

According to Shafer's approach and unlike Smets' rule, Dempster-Shafer (DS) rule does not allow the attribution of belief mass to the conflict. Thus, in DS, we get:

$$
m_{1,2}^{D S}(\varnothing)=0
$$

The conflict mass is then reallocated through a normalization factor. The mass of belief in $A, m_{1,2}^{D S}(A)$, resulting from the fusion from two information sources is written:

$$
m_{1,2}^{D S}(A)=\frac{1}{1-m_{1,2}(\varnothing)} \sum_{X, Y \subseteq \Theta \mid X \cap Y=A} m_{1}(X) m_{2}(Y)
$$

\subsection{Geophysical belief masses distribution}

To attribute belief masses from electrical resistivity and shear wave velocity data to each cell of the sections (ERT and MASW discretized sections respectively), it is first necessary to define the limits of the resistivity and velocity intervals corresponding to the hypotheses of the FoD. We use a $K$-means clustering method (Likas et al. 2003 ) to divide the geophysical parameters into $K$ clusters.

In Figure 2, three sets of electrical resistivities emerge. We suggest to associate low values with $\theta_{1} \cup \theta_{2}$ (conductive materials: fine-grained fill or marls) and high values with $\theta_{3} \cup \theta_{4}$ (resistive materials: coarse-gained fill or limestones). Because the intermediate values do not give information on the precise nature of the lithological material, they are associated with $\theta_{1} \cup \theta_{2} \cup \theta_{3} \cup \theta_{4}$. Thus, we consider three clusters for the use of K-means clustering classification and get in $\Omega . \mathrm{m}$ :

[2,5; 75] associated with $\theta_{1} \cup \theta_{2}$, ]75; 354[ associated with $\theta_{1} \cup \theta_{2} \cup \theta_{3} \cup \theta_{4}$, $\left[354 ; 10^{4}\right]$ associated with $\theta_{3} \cup \theta_{4}$.

For the MASW method, three sets of velocities emerge as well. We associate low velocities with finer materials $\theta_{1} \cup \theta_{2}$ and high velocities with coarser materials $\theta_{3} \cup \theta_{4}$. The intermediate values of $V s$ are associated with $\theta_{1} \cup$ $\theta_{2} \cup \theta_{3} \cup \theta_{4}$. Thus, we consider three clusters for the use of K-means clustering classification and get in $\mathrm{m} \cdot \mathrm{s}^{-1}$ :

[180; 450] associated with $\theta_{1} \cup \theta_{2}$, ]450; 670[ associated with $\theta_{1} \cup \theta_{2} \cup \theta_{3} \cup \theta_{4}$, $\left[670 ; 1,3.10^{3}\right]$ associated with $\theta_{3} \cup \theta_{4}$.

Once the hypotheses of the FoD have been characterized for each information source (here in $\Omega$.m and in $\mathrm{m} \cdot \mathrm{s}^{-1}$ respectively), belief masses must be assigned to each hypothesis of $2^{\Theta}$. This has to be done for each cell of the section and for each information source. The belief masses are computed as a function of the "distance" between the inverted values in each cell and the intervals associated with the FoD hypotheses (Tran and Duckstein, 2002). Thus, the more the distance for an inverted value is "close" to a hypothesis of the FoD, the more the associated belief mass is and reciprocally. The procedure takes into consideration the imprecisions associated with the geophysical parameter values and the sensitivity of the methods. It is detailed in Chapter II.B, Section 3.4.4 of Dezert (2019) as well as in Dezert et al. (2020). The sensitivity of the methods and the reliability of the inverse models are important questions that are not trivial and that we will not deal with in this work. Each cell of the section is then associated with belief masses such that the sum of the masses is equal to 1 (Eq. 4).

The representations of this approach are displayed Figure 5 and Figure 6 for the ERT and MASW methods respectively. Figures 5.a and 6.a highlight the hypotheses 


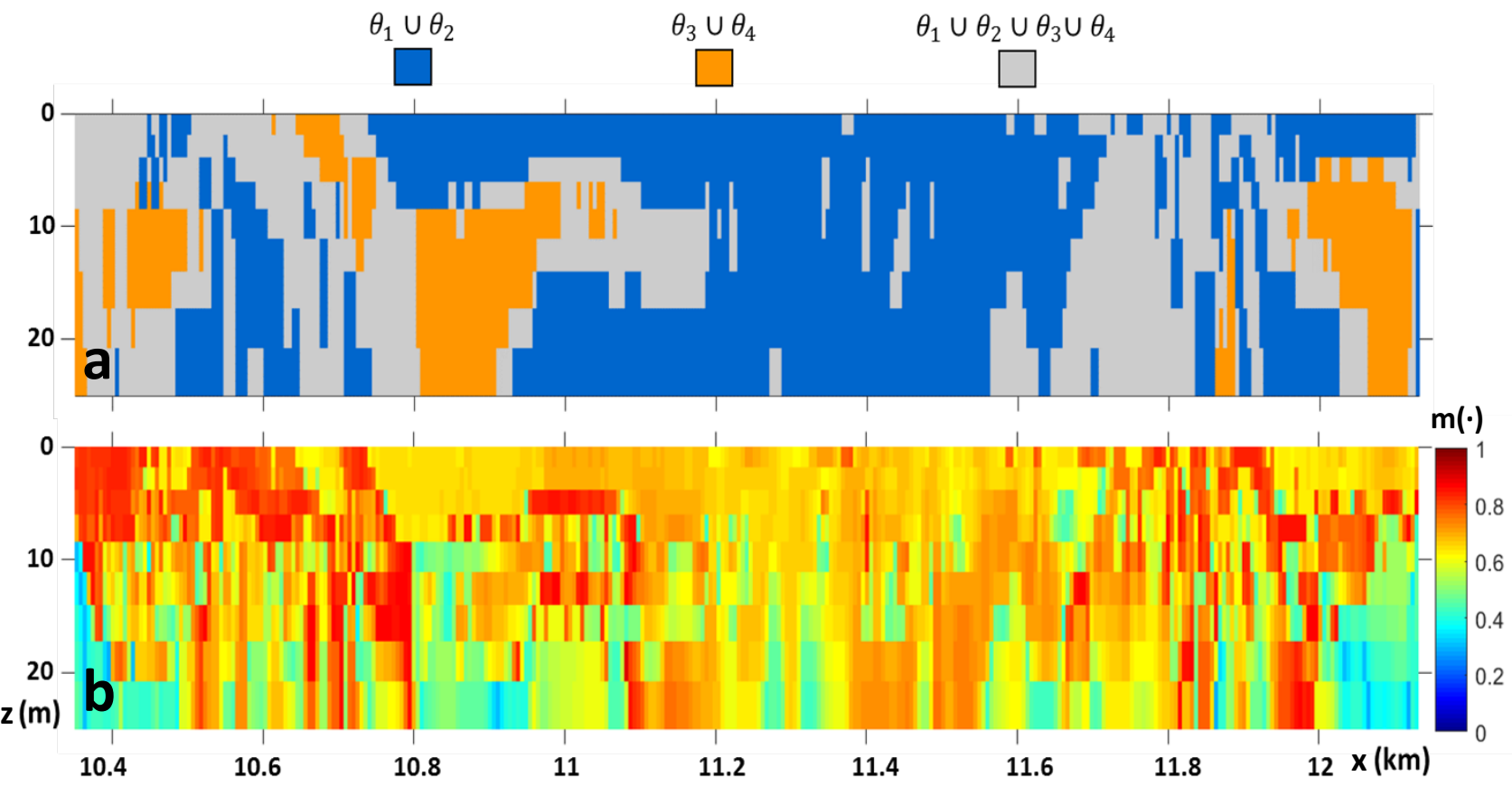

Figure 5. a) Hypothesis having the highest belief mass for the ERT characterization, b) associated belief masses.

having the greatest belief mass for each cell while Figures 5.b and 6.b display their associated belief mass values. The representations of these results show the ability of our methodology to represent the uncertainty (representation of a union of hypotheses with associated confidence indexes).

The four areas of high resistivities present in Figure 2 are depicted in orange in Figure 5.a. Figure 5.b shows that the lowest belief masses are located at the bottom of the section as well as on the sides. This is attributed to the high values of inaccuracies associated with the resistivities in these areas of the section. Figures 5 and 6 show that the ERT and MASW characterizations of the dike do not make it possible to individually dissociate the four hypotheses (fine fill, coarse fill, marls and limestones) since these materials may have the same resistivity/velocity values.

Figure 6 highlights the ability of the methodology to represent the lack of information (incompleteness). Indeed, where there is no information concerning the shear wave velocities, the belief mass is set to 1 (in red, Figure 6.b) on the union of all hypotheses, $\theta_{1} \cup \theta_{2} \cup \theta_{3} \cup \theta_{4} \cup$ $\theta_{5}$ (in black, Figure 6.a). It appears that these results are rather in agreement with the characterization proposed by the ERT method Figure 5, in particular with the characterization of $\theta_{1} \cup \theta_{2}$ at shallow depth for the three sections covered and the characterization of $\theta_{3} \cup \theta_{4}$ below $10 \mathrm{~m}$ depth for the first section, around KP 10.8.

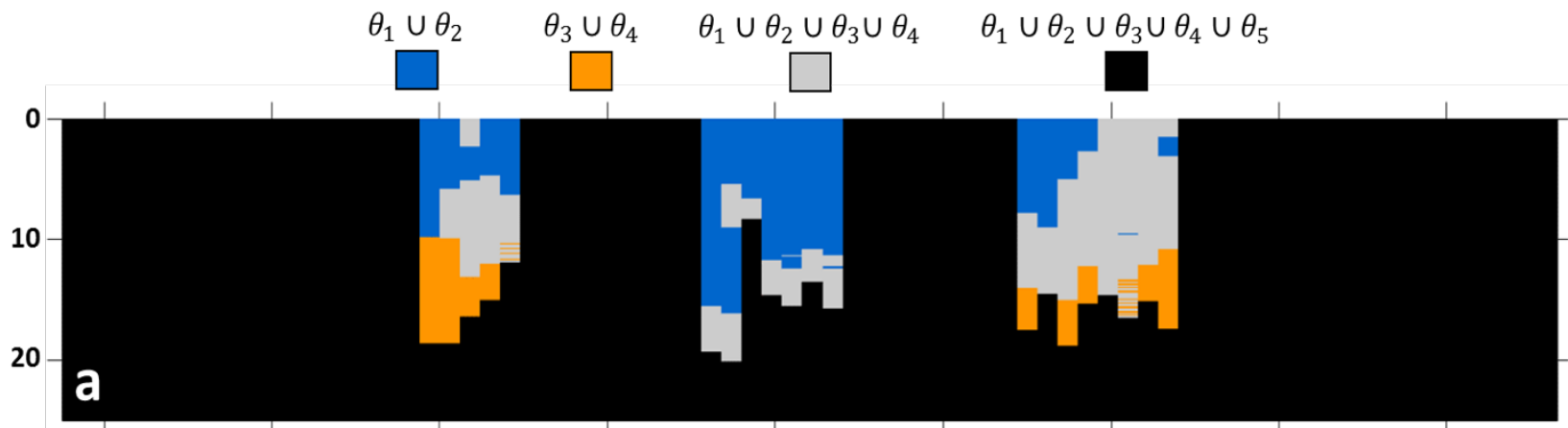

$\mathrm{m}(\cdot)$

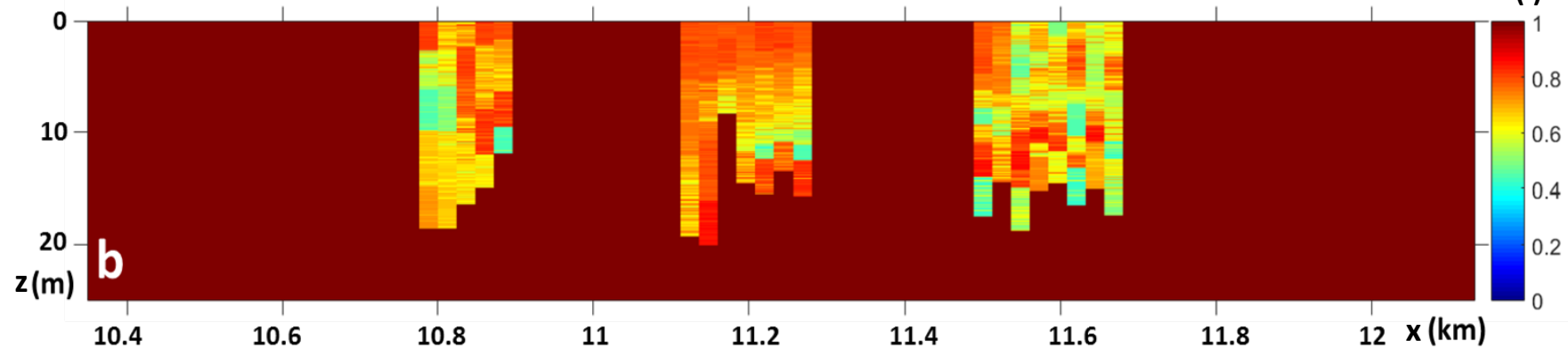

Figure 6. a) Hypothesis having the highest belief mass for the MASW characterization, b) associated belief masses. 


\subsection{Geotechnical belief masses distribution}

As for the two geophysical methods, it is necessary to be able, for each cell of the geotechnical discretized section, to associate particle-size analysis data or geotechnician observation with the hypotheses of the FoD. Unlike the ERT and MASW methods, the extracted materials allow us to discriminate the four hypotheses individually: $\theta_{1}, \theta_{2}, \theta_{3}$ and $\theta_{4}$.

A belief mass close to $1, m(\cdot)=0.99$, associated with the characterized hypothesis is fixed in the cells at the borehole positions. Complementarily, a mass of $m(\cdot)=$ 0.01 is then associated with the union of all hypotheses $\left(\theta_{1} \cup \theta_{2} \cup \theta_{3} \cup \theta_{4} \cup \theta_{5}\right)$, in agreement with Eq (4). The belief masses are set to 0 on the remaining hypothesis.

The belief mass of 0.99 can theoretically be modified according to the ability of the geotechnical method to characterize the investigated material. However, we believe that the extraction of materials and their observations as well as particle analysis is the most reliable way to characterize the dike's lithology, justifying the chosen level of confidence in the boreholes.

We then construct a geotechnical discretized mesh, covering the complete section of the dike. In order to characterize the entire section located within the ERT profile, and to associate belief mass values to each newly generated cell, we impose an exponential lateral decay of the belief mass from the borehole point to the nearby one so that the decay rate is a function of the values proposed by the nearby borehole. Thus, we get for a specific depth:

$$
\mathrm{M}(\mathrm{x})=0.99 \cdot e^{-k C_{v} x}
$$

With $x$ being the horizontal distance from the considered cell to the reference borehole in meters (with $x=0$ in the borehole), $M(x)$ the belief mass values assigned to each hypothesis of the FoD for a position $x, k$ a lateral decay coefficient and $C_{v}$ the coefficient of variation of particle-size values.

The coefficient $k$ value is chosen by the user and is a function on the lateral variability of the investigated terrain. A parametric study on the influence of this coefficient can be found in Dezert et al. (2019). Here, such as in Dezert et al. (2019), we consider a value of $k=0.1$. The expression of $C_{v}$ is written such that:

$$
C_{v}=\sqrt{\frac{1}{n_{m e s h}-1} \sum_{i=1}^{n_{m e s h}}\left(Q-Q_{i}\right)^{2}}
$$

Where $Q$ is the grain-size parameter value of the considered cell in the borehole (cumulative sieve under 80 $\mu \mathrm{m})$ and $Q_{i}$ the value in the nearby borehole centred on the same depth. Here, we set $n_{m e s h}=7$, so that the computation of $C_{v}$ takes into account 7 meshes in the nearby borehole (i.e. thickness of $70 \mathrm{~cm}$ ).

This justifies why boreholes B1 and B7 are used in this work, although they are not located within the ERT profile (Figure 4). Indeed, they make it possible to compute the coefficients of variation under concern: they enable the computation of the decay rate of B2 belief masses to the left and the decay rate of B6 belief masses to the right. For a given cell in the section, when the belief mass associated with a hypothesis is lower than 1, the remaining of the mass to be assigned is allocated to the hypothesis "any material" $\left(\theta_{1} \cup \theta_{2} \cup \theta_{3} \cup \theta_{4} \cup \theta_{5}\right)$. Beyond the maximum depth of geotechnical investigations, it is considered that $m\left(\theta_{1} \cup \theta_{2} \cup \theta_{3} \cup \theta_{4} \cup \theta_{5}\right)=1$, since no information is available.

The lithological materials most likely to be in the dike section according to the drilling cores are displayed in Figure 7.a with their associated belief masses in Figure 7.b. This figure highlights the great confidence (high belief

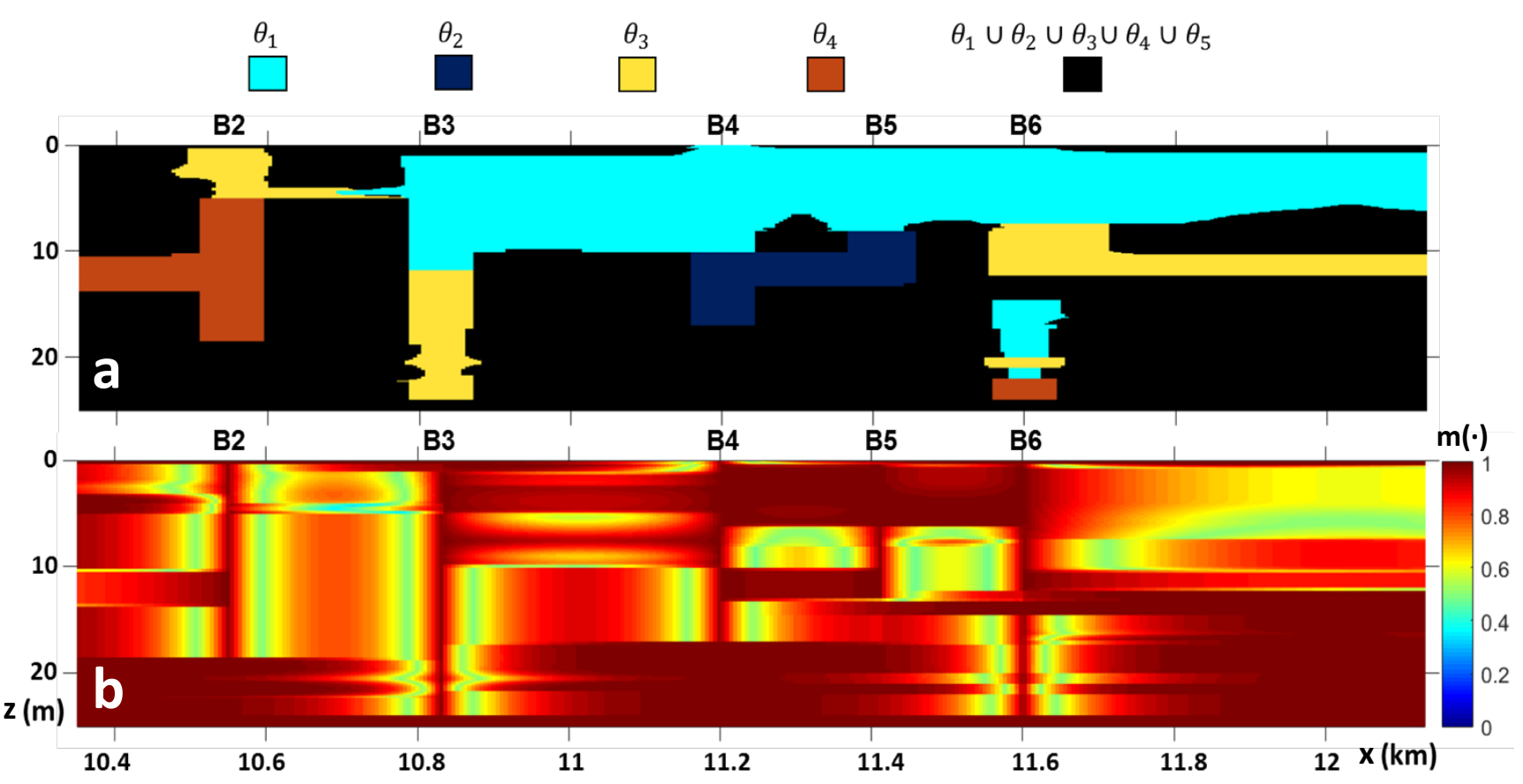

Figure 7. a) Hypothesis having the highest belief mass for the core drilling characterization, b) associated belief masses. 
mass values) close to the borehole positions as well as the variable lateral decay rates, which are a function of the materials characterized in the nearby boreholes. For example, considering borehole B4 (Figure 7): the confidence associated with the hypothesis $\theta_{1}$ (fine fill materials) extends largely to the right over the first 8 meters because the nearby borehole (B5) characterizes the same lithological material at these depths. The extent of confidence in this hypothesis is much more restricted at greater depths (between $\mathrm{z}=8$ and $\mathrm{z}=10 \mathrm{~m}$ ), since another hypothesis $\left(\theta_{2}\right.$, marls $)$ is characterized in B5 at these depths. As for the MASW method, these figures highlight the capacity of our methodology to display the lack of information (incompleteness). Indeed, the complete uncertainty $\left(\theta_{1} \cup \theta_{2} \cup \theta_{3} \cup \theta_{4} \cup \theta_{5}\right)$ is displayed in black in Figure 7.a.

\section{Results}

Even though the fusion procedure can be carried out between the three information sources, we display only the fusion results considering ERT and MASW methods on one hand (Figure 8) and considering ERT and core drilling
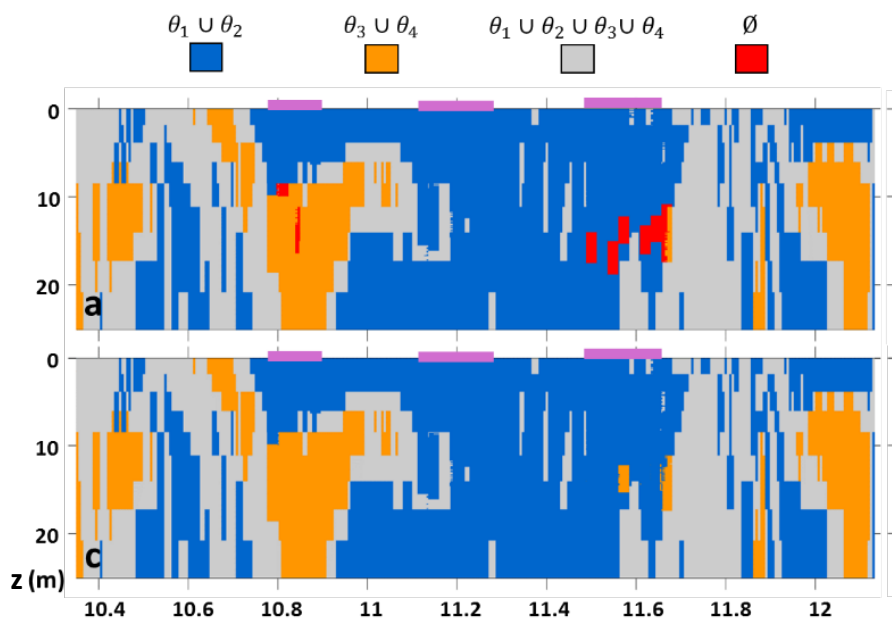

methods on the other hand (Figure 9). This choice has been made since the conflict level between the MASW and ERT methods is very low, as depicted in Figure 8.

\subsection{ERT and MASW fusion results}

Since neither of these two methods are able to discriminate individually the four lithological materials, these fusion results only display the union of hypotheses (Figure 8.a and 8.c). The associated belief masses are respectively displayed Figures 8.b and 8.d. While Figures 8.a and 8.b stand for the fusion results using Smets' combination rule, Figures 8.c and 8.d stand for the fusion results using the DS combination rule.

Figures 8.b and 8.d highlight that the confidence is enhanced when the same lithological materials are characterized by both investigation methods, especially near the dike crest when fine fill or marl materials are characterized. Two small conflict areas appear. The largest one is situated below the $3^{\text {rd }}$ section of MASW (KP 11.5$11.7)$ at $15 \mathrm{~m}$ depth. While the MASW method characterizes the $\theta_{3} \cup \theta_{4}$ hypothesis (Figure 6.a), the ERT method characterizes the $\theta_{1} \cup \theta_{2}$ hypothesis (Figure 5.a). Globally, the characterizations proposed here are very
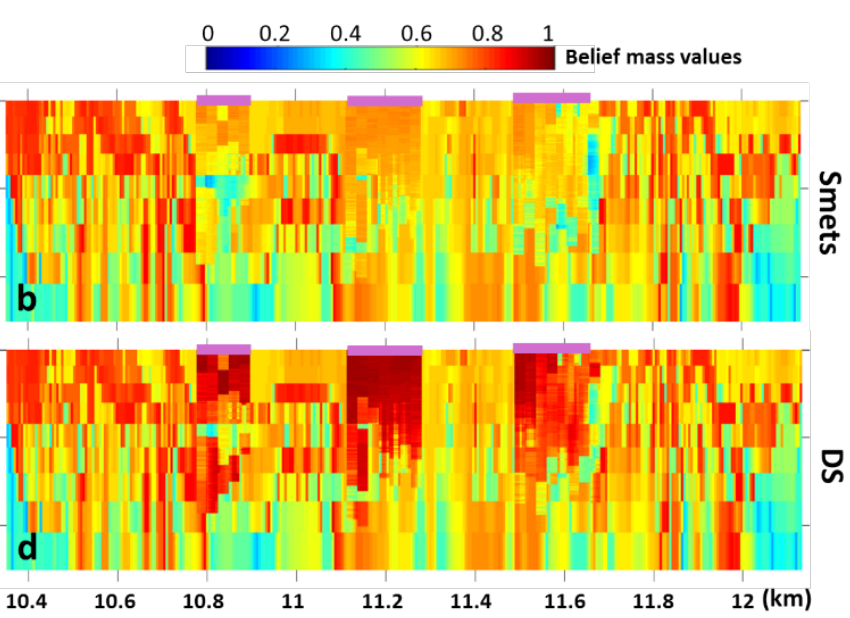

Figure 8. a, c) Hypothesis having the highest belief mass according to the masses attribution from ERT and MASW data fusion with the Smets and DS rules, b, d) associated belief masses for each rule. Purple lines stand for the position of the MASW profiles.
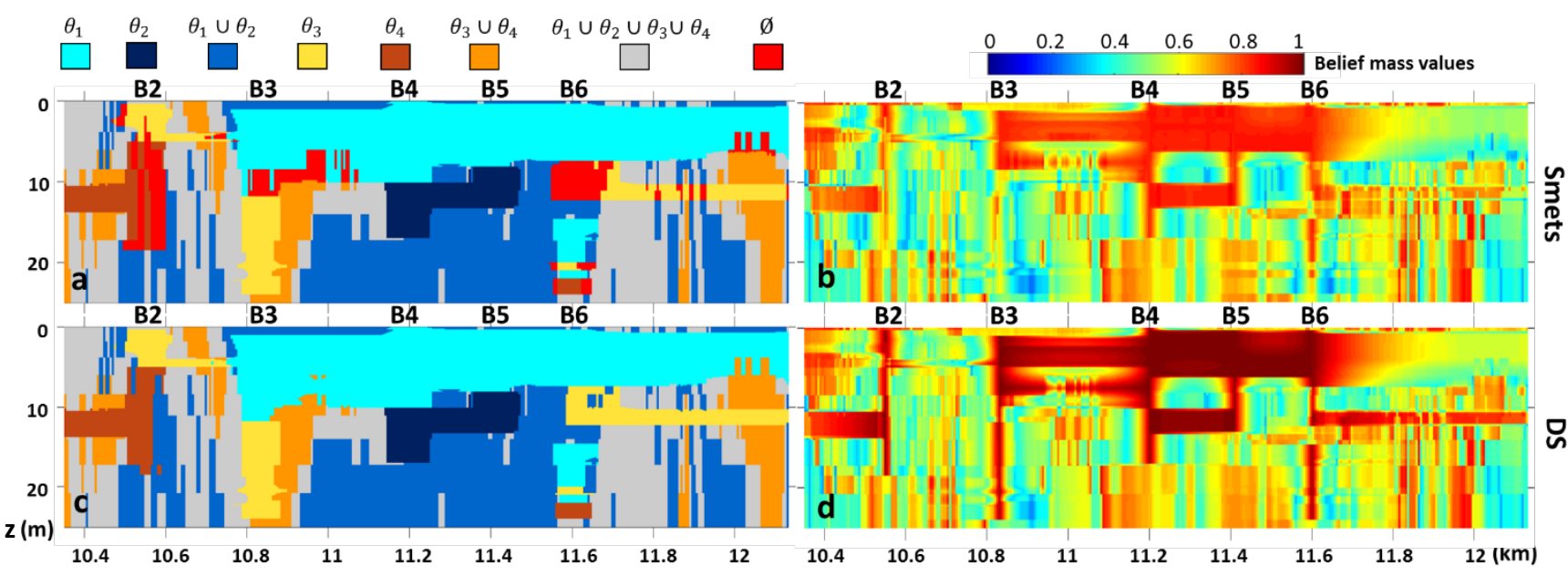

Figure 9. a, c) Hypothesis having the highest belief mass according to the masses attribution from ERT and core drilling data fusion with Smets and DS rules, b, d) associated belief masses for the Smets and DS rules respectively. 
close to the one made by the ERT alone (Figure 5) since it provides information on a much broader area than the MASW method. Indeed, a large part of the dike is characterized with $m\left(\theta_{1} \cup \theta_{2} \cup \theta_{3} \cup \theta_{4} \cup \theta_{5}\right)=1$ for the MASW method (in black, Figure 6.a) which does not provide any information during the fusion process.

\subsection{ERT and drilling core data fusion results}

The fusion results from the ERT and core drilling methods are displayed in Figure 9. Compared to the geophysical fusion results displayed in the previous section, the contribution of core drillings is important since they allow to discriminate the lithological materials individually and to display precise positions of interfaces.

Thanks to Smets' combination rule, it can be noted in Figures 9.a and 9.c that the conflict level decreases when deviating from the borehole positions since the confidence level on the geotechnical information decreases with the distance to these geotechnical testing points. Comparatively, the influence of the ERT characterization becomes gradually more important.

\section{Discussion}

The obtained ERT and MASW fusion results (Figure 8) justify why we do not keep the MASW characterization when including drilling core data (Figure 9): i) the MASW method covers much narrower areas than the ERT, ii) it cannot discriminate the materials individually, iii) the conflict level between the two geophysical methods is very low (Figure 8.a). Indeed, the MASW characterization mainly confirms the ERT characterization without bringing new significant information.

The belief masses of the characterized lithological materials seem lower with ERT and drilling core data (Figures 9.b and 9.d) than with ERT and MASW methods (Figures 8.b and 8.d). However, it does not mean that these fusion results are of lower quality. Indeed, it must be highlighted that individual materials are locally characterized (Figures 9.a, 9.c) while in Figure 8 (ERT and MASW fusion results) only union of materials are characterized. This characterization is therefore more precise than what was proposed by considering only the ERT and MASW methods. The zones where conflict is present with Smets' rule are zones where the confidence is low with the DS rule (e.g. KP 10.8 - 11 at 10 meters depth, conflict represented in Figure 9.a and associated low masses after use of the DS rule, Figure 9.d).

The fusion results obtained are compatible with the (low resolution) lithological information that was available on the dike before the use of our methodology. Actually, Figure 9.c proposes the presence of limestone materials up to KP 10.6 with coarse fill materials above. Beyond, the area between KP 10.6 and KP 10.8 is poorly characterized: the union of four hypotheses is mainly depicted in Figure 9.c with low associated belief masses (Figure 9.d). This most probably corresponds to the NE-SW fault lowering the western compartment that was known to be present. Indeed, it makes sense for a very heterogeneous area to be poorly characterized. This also explains why coarse fill materials are present at such depths at KP 10.8 while they are closer to the dike crest at KP 10.55 .

In addition, we know from historical sources that the KP 10.8 area was the subject of a specific treatment of the foundation because of the presence of compressible ground in the foundation ("peat" and "blue vases") which had caused settlements and cracks in the initial dike. These lands have been completely replaced by coarse-fill materials (about 8 meters deep below the old backfillfoundation limit). This explains the presence of the resistive lens of the electric acquisition between KP 10.8 and KP 10.95.

Further, fine fill materials seem present up to about 10 $\mathrm{m}$ depth with marl basement below. From KP 11.6, the lithological characterization is less manifest with probably the alternation of fine and coarse fill materials. Geophysical uncertainty (Figure 5) as well as the lack of geotechnical characterization (Figure 7) leaves the section below $10 \mathrm{~m}$ deep rather unclear. The available knowledge suggests that limestone materials could be present between KP 11.5 and KP 12.13, which seems consistent with the fusion characterizations depicted at the end of the section (Figure 9.c).

Globally, the obtained results display more precise lithological interface positions than what each method could propose individually. Complementarily to the capacity to characterize lithological materials individually (thanks to core drillings), it is of interest to have areas where the uncertainty is displayed between two or even four materials. This information could be precious to know where the geotechnical investigations could be strengthened.

Where the ERT investigation method is unable to discriminate the four hypotheses (Figure 5), the geotechnical data allow the section to be more precisely characterized by discriminating the lithology and by specifying the position of the interfaces between fine and coarse fill materials (e.g. at about $11.5 \mathrm{~m}$ depth, KP 10.8). Our fusion methodology enables to display conflict between information sources thanks to Smets' combination rule (Figures 8.a and 9.a). This notion of conflict is precious and to our knowledge, there is no other work displaying this type of information.

Because conflict is not a lithological solution, the DS combination rule is also interesting. It brings out the lithological material most likely present regardless of the high conflict level. The belief masses associated to the characterizations are also of important interest (Figure 8.b, 8.d, 9.b and 9.d). Even though these belief masses should not be judged as absolute indicators, they provide precious information when they are used jointly, in a relative way.

In some locations, the union of two hypotheses is depicted after the fusion process (e.g. $\theta_{1} \cup \theta_{2}$ below $13 \mathrm{~m}$ depth at KP 11.4). However, an expert's observation should be sufficient to suggest the most plausible solution. For this precise example, although B5 core drilling does not go further than $13 \mathrm{~m}$ deep, it seems reasonable that the materials located below are also marls $\left(\theta_{2}\right)$ and not fine fill materials $\left(\theta_{1}\right)$.

Finally, an expert's interpretation is important to use this methodology in order to propose the correct number $\mathrm{K}$ of clusters. Here, three clusters are used for the ERT and 
the MASW methods, but other geophysical surveys may require a different number of clusters. It is also the expert's duty to know which lithological material to associate to each cluster, according to their knowledge of the investigated site. At present time, it is still one of the limitations of this method since it is not fully automated.

\section{Conclusion}

In this work, we introduced an innovative fusion methodology based on the use of belief masses to combine data from geophysical (ERT and MASW) and geotechnical investigation methods (core drilling with particle-size analysis). This methodology has been applied to a canal dike characterization. These information sources are judged complementary, each one having its own spatial distribution and associated level of imperfections. We have compared the fusion results with two combination rules (Smets and DS) for ERT and MASW data fusion as well as for ERT and core drilling data fusion. A representation of the merged information associated with degrees of belief has been proposed.

The fusion results highlight the important variability in lithology in the studied section as well as the presence of a known fault within the area. They also provide information on the location of fine and coarse fill materials, limestone and marls. The suggested graphical representations of the results make possible to locate areas of strong confidence (important belief masses), of doubt between two or four lithological materials (union of hypotheses) and of conflict between information sources (high belief masses associated with $\emptyset$ ). These results also depict consistence between the characterization made by the ERT and the MASW methods.

Finally, in the fusion results, the zones of lesser confidence level indicate where the investigations could be reinforced. Moreover, the conflict areas inform where two information sources disagree. These two types of information may be precious to carry out an investigation campaign as well as for decision support (e.g., models of failure hazards). This methodology would also be appropriate to other applications (quantification of liquefaction risk, pedology, landslide, pollutant tracking, mining...) when at least two information sources are involved.

\section{References}

1. AFNOR NF P11-300 (1992). Exécution des terrassements - Classification des matériaux utilisables dans la construction des remblais et des couches de forme d'infrastructures routières.

2. AFNOR NF P94-056 (1995). Sols : reconnaissance et essais - Analyse granulométrique - Méthode par tamisage à sec après lavage.

3. Dempster, A.,P. (1967). Upper and lower probabilities induced by a multivalued mapping. The annals of mathematical statistics, Springer, Berlin, pp 325-339.

4. Dezert, T. (2019). Combinaison d'informations ponctuelles et volumiques pour le diagnostic d'ouvrages en terre soumis à des risques hydrauliques
(Doctoral dissertation, Université de Nantes-Faculté des Sciences et Techniques), 242 p, tel-02459100.

5. Dezert, T., S.P. Lopes, Y. Fargier and P. Côte (2020). Data Fusion of In-Situ Geophysical and Geotechnical Information for Dike Characterization. Bulletin of Engineering Geology and the Environment.

6. Fauchard C. and P. Mériaux (2007). Geophysical and geotechnical methods for diagnosing flood protection dikes: Guide for implementation and interpretation. Quae.

7. Likas, A., N. Vlassis and J.J. Verbeek (2003). The global k-means clustering algorithm. Pattern recognition, 36(2): 451-461.

8. Loke, M.H. (2013). Tutorial: 2-D and 3-D electrical imaging surveys. http://www.geotomosoft.com.

9. Martin, A., C. Osswald, J. Dezert and F. Smarandache (2008). General combination rules for qualitative and quantitative beliefs. Infinite Study.

10. Royet P, S. Palma Lopes, C. Fauchard, P. Mériaux and L. Auriau (2013). Rapid and Cost-Effective Dike Condition Assessment Methods: Geophysics and Remote Sensing. FloodProBE FP7 European Project.

11. Tran L. and L. Duckstein (2002). Comparison of fuzzy numbers using a fuzzy distance measure. Fuzzy sets and systems, 130(3), 331-341. 